\title{
Rate improvements and power saving with a hybrid filtered multitone scheme
}

\author{
Salvatore D'Alessandro and Andrea M Tonello*
}

\begin{abstract}
This article presents a hybrid adaptive multi-carrier architecture that, according to the channel condition, wisely switches between orthogonal frequency division multiplexing (OFDM) and orthogonal filtered multitone (FMT) modulations. This new scheme is referred to as hybrid-FMT (H-FMT). For H-FMT, the problem of jointly allocating the sub-channels powers, the overhead duration, and the modulation scheme, to the channel condition to (a) maximize the achievable rate, or (b) minimize the total transmitted power, is considered. Furthermore, we present an efficient implementation that requires minimal increased complexity w.r.t. baseline OFDM. The potential benefits given by the adoption of H-FMT are shown through extensive numerical results over typical WLAN channels.
\end{abstract}

Keywords: OFDM, FMT, Resource allocation, Hybrid modulation, Green communication

\section{Introduction}

Power saving is playing an important role in the development of advanced communication technologies. In fact, energy efficiency and saving are fundamental objectives to contribute to the Sustainable Growth also specified in the "Europe 2020" strategy. However, not only power saving, but also high bit-rate has to be granted for those applications that need high quality of service. For example, considering wireless local area networks (WLANs) or personal area networks scenarios, the increasing demand for high-quality multimedia services to be offered in consumer electronics devices has pushed the development of new communication technologies that make use of advanced communication techniques such as multi-carrier (MC) modulation, cooperative communication [1], resource allocation, and cross-layer optimization [2]. Some relevant examples are the devices compliant with the $802.11 \mathrm{n}$ or $802.15 .3 \mathrm{c}$ IEEE wireless standards.

This article proposes the use of a novel hybrid MC architecture that allows for (a) improving the achievable rate, or for (b) saving power w.r.t. conventional orthogonal frequency division multiplexing (OFDM) for the WLAN application scenario. The scheme is referred to as hybrid filtered multitone (H-FMT) [3]. Depending on

\footnotetext{
* Correspondence: tonello@uniud.it

Dipartimento di Ingegneria Elettrica, Gestionale e Meccanica (DIEGM),
} Università di Udine, Udine 33100, Italy the channel condition, H-FMT switches between filtered multitone (FMT) modulation with minimal length orthogonal pulses and adaptive-OFDM (A-OFDM). To this end, two switching criteria that allow H-FMT to select the modulation are jointly designed with the resource allocation algorithms to maximize the achievable rate or to minimize the transmitted power.

FMT is a discrete-time implementation of MC modulation that uses uniformly spaced sub-carriers and identical sub-channel pulses. It has originally been proposed for application over broadband wireline channels [4], and subsequently it has been investigated for applications over wireless channels [5,6]. The considered FMT deploys orthogonal filters with minimal length equal to the duration of one transmitted symbol. This renders the system to be interference free in ideal conditions and makes the filters to be maximally confined in frequency. Consequently, when signaling over a frequency selective channel both the inter-carrier interference (ICI) and the inter-symbol interference (ISI) are well mitigated. Furthermore, its computational complexity is comparable to that of OFDM [7].

OFDM can be seen as an FMT that uses rectangular sub-channel pulses. It is well known that in presence of a frequency selective channel, the cyclic prefix (CP) is needed to mitigate the interference components. If the $\mathrm{CP}$ is longer than the channel impulse response, the system maintains its perfect reconstruction property, thus, 
no interference is present at the decision stage. Clearly, when the CP is shorter than the channel duration, ISI and ICI terms arise [8]. In [9], it was found that over WLAN channels, the CP has not to be necessarily as long as the channel duration to maximize the achievable rate. The rationale behind it is that the interference can be increased in noise-limited systems without affecting the achievable rate. Furthermore, a nearly optimal value of $\mathrm{CP}$-designed according to the statistic of the achievable rate optimal CP duration-was found for each channel class of the IEEE 802.11n WLAN channel model [10]. The OFDM that adapts the CP to the channel condition is referred to as A-ODFM.

Thanks to the similarities of the efficient implementation of both FMT with minimal length pulses and AOFDM [11], the realization of H-FMT introduces a marginal increase in computational complexity w.r.t. conventional OFDM. This renders the proposed scheme suitable for being implemented in consumer electronics devices that offer WLAN connectivity, e.g., notebooks, tablets, or smart phones.

This article first focuses on the achievable rate maximization (RM) problem of H-FMT. To this end, two switching criteria that allow H-FMT to select the modulation, namely FMT or A-OFDM, that maximizes the achievable rate are presented. The first switching criterion is optimal but characterized by a high computational complexity. The second is sub-optimal but has low complexity inasmuch it selects the modulation based on the estimation of the signal-to-noise ratio (SNR). Afterwards, the power minimization (PM) problem for H-FMT is considered. Since H-FMT is based on A-OFDM and FMT, in general, it suffers from interference. This makes the PM problem not convex, and therefore hard to solve. To simplify the problem, an iterative algorithm is proposed. At each iteration, it assumes the interference to be independent on the distribution of the transmitted power, and thus it solves the corresponding convex PM problem using the KarushKuhn-Tucker (KKT) conditions [12].

Extensive numerical results over typical WLAN channels compare H-FMT with conventional OFDM that uses a conservative fixed value of $\mathrm{CP}$. They show that H-FMT may significantly increase the achievable rate of WLAN devices w.r.t. OFDM, without increasing the transmitted power, and further, it outperforms OFDM up to $3 \mathrm{~dB}$ in terms of transmitted power-yet reaching the same rate.

The remainder of this article is organized as follows. In Section 2, the general MC system model as a filter bank scheme is introduced, and OFDM and FMT are derived. The RM problem is presented in Section 3, and it is applied to H-FMT in Section 4. Some of the results obtained when solving the RM problem of H-FMT are used in Section 5, to solve the dual problem, namely, the PM problem of H-FMT. Section 6 presents the numerical results, and finally, the conclusions follow in Section 7.

\section{System model}

A complex baseband MC modulation scheme is considered (see Figure 1). In MC modulations, a high data rate signal is split into $M$ parallel signals $a^{(k)}(\ell N)$, where $k$ is the subchannel index $(k=\{0, \ldots, \mathrm{M}-1\}), N$ is the symbol period in samples, and $\ell$ is the time index. The symbol period in seconds is given by $N T$, where $T$ is the sampling period ${ }^{\mathrm{a}}$.

The parallel signals, which in general are streams of data symbols belonging to a complex constellation, e.g., M-ary QAM, are interpolated by a factor $N$, filtered by the subchannel pulses $g^{(k)}(n)=g(n) e^{j \frac{2 \pi k n}{M}, k},\{0, \ldots, M-1\}$ (which are the modulated version of the prototype pulse $g(n))$, summed and transmitted over the channel. The transmitted signal can be therefore expressed as the output of a synthesis filter-bank (FB), i.e.,

$$
x(n)=\sum_{k=0}^{M-1} \sum_{\ell \in \mathbb{Z}} a^{(k)}(N \ell) g^{(k)}(n-N \ell) .
$$

In (1), the interpolation factor $N$ is equal to $M+\beta$ samples, where $\beta$ denotes the overhead $(\mathrm{OH})$ factor. The channel is modeled as a linear time-invariant filter. In particular, the IEEE 802.11 task group $\mathrm{n}$ (TGn) channel model [10] is adopted. This model was developed within TGn for indoor WLAN systems. It allows for generating channels belonging to five classes, which are labeled with B,C,D,E,F and are, respectively, characterized by a given average rms delay spread, i.e., $\{25,39$, $55,100,150\}$ ns. Furthermore, each class is a representative of a certain environment, e.g., classes B and C are representatives of residential homes and small offices, class D of typical office environments, classes $\mathrm{E}$ and $\mathrm{F}$ of a large indoor open space/office. Both line of sight (LOS) and non-LOS (NLOS) propagation are modeled. This model also accounts for small-scale multipath fading and large-scale path loss fading as a function of distance. A detailed description of the model is reported in [10] and in the references therein.

The continuous time complex impulse response provided by such a model can be written as

$$
\hat{g}_{\mathrm{ch}}(t, d)=A(d) \sum_{p=0}^{v-1} \alpha_{p} \delta\left(t-\tau_{p}\right),
$$

where $A(d)$ is the attenuation attributed to the path-loss when the transmitter and the receiver are at distance $d$. The number of multipath components is denoted by $v$. Depending on the distance and on the channel class the path-loss is either computed using deterministic 


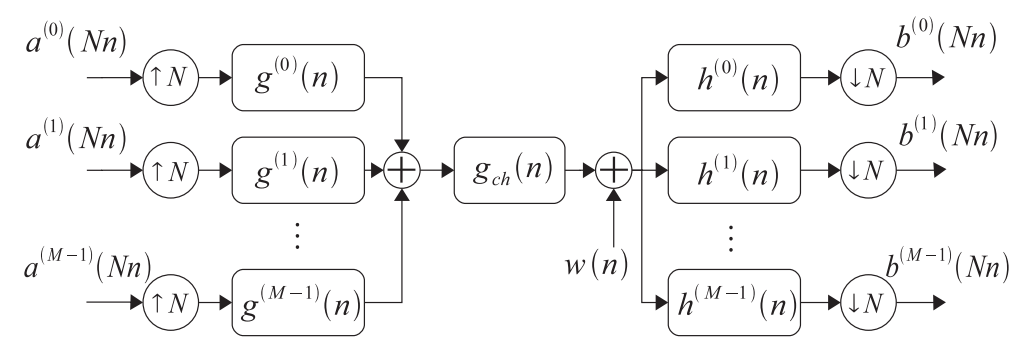

Figure 1 General MC architecture as a filter bank scheme.

models or it is drawn from a log-normal shadow fading distribution. $\alpha_{p}$ denote the complex channel coefficients whose amplitude can be Rayleigh or Rician distributed depending on the channel class and the associated time delay $\tau_{p}$. A low pass filter, before sampling the signal with frequency $B=1 / T$, is used to obtain the equivalent discrete time channel impulse response. Additive white Gaussian noise (AWGN) is assumed. Therefore, the received signal, denoted by $y(n)$, is processed by an analysis FB whose $k$ th sub-channel output is

$$
\begin{aligned}
b^{(k)}(N n) & =\sum_{m \in \mathbb{Z}} y(m) h^{(k)}(N n-m) \\
& =U^{(k)}(N n, \beta)+I^{(k)}(N n, \beta)+\eta^{(k)}(N n, \beta) .
\end{aligned}
$$

According to (3), the signal $y(n)$ is filtered by the modulated filter $h^{(k)}(n)=h(n) e^{j \frac{2 \pi}{M} k n}$, where $h(n)$ is the prototype pulse, and down-sampled by a factor $N$. Furthermore, the received signal in the $k$ th sub-channel can be decomposed into three components: the signal of interest $U^{(k)}(N n, \beta)=$ $a^{(k)}(N n) H^{(k)}(\beta)$, the interference $I^{(k)}(N n, \beta)$ consisting of ISI and ICI, and the noise component $\eta^{(k)}(N n, \beta)$. The channel transfer function at the center frequency of sub-channel $k$ is defined as $H^{(k)}(\beta)=g^{(k) *} g_{c h}{ }^{*} h^{(k)}(0)$. These components are in general all dependent on the $\mathrm{OH}$ factor $\beta$ as shown in (3).

\subsection{OFDM}

The OFDM system can be obtained from the general $\mathrm{MC}$ scheme setting the synthesis and the analysis prototype pulses, respectively, equal to

$$
g(n)=\operatorname{rect}(n / N), \quad h(n)=\operatorname{rect}(-n / M),
$$

where $\operatorname{rect}(n / N)=1$ for $n=\{0,1, \ldots, N-1\}$ and zero otherwise.

The $\mathrm{OH}$ factor $\beta=N-M$ corresponds to the $\mathrm{CP}$ duration in number of samples. The $\mathrm{CP}$ copes with the interference caused by the transmission over a dispersive channel. In general, the design of the $\mathrm{CP}$ duration in
OFDM systems consists in choosing a certain value of $\mathrm{CP}$ that results longer than most of the channel durations such that no interference occurs at the receiver side [8]. However, this benefit is paid in term of (a) a loss in SNR of a factor $\beta /(M+\beta)$ since the prototype pulses are not matched, and as it will be shown in the following, (b) a loss in achievable rate of a multiplicative factor $M /(M+\beta)$.

\subsection{FMT with minimal length pulses}

Different from OFDM, in FMT the analysis pulse is matched to the synthesis pulse, i.e., $h(n)=g^{*}(-n)$. A distinctive characteristic of FMT is that the prototype pulse is designed to obtain high-frequency confinement [4], namely long prototype pulses allow FMT to experience a negligible ICI term. Therefore, the equalization task focuses on canceling the ISI term by means of linear sub-channel equalizers. In general, FMT with long prototype pulses performs better than OFDM, although its implementation complexity is higher [13].

In this article, to lower the implementation complexity of FMT and to make use of an efficient implementation realization with minimal changes w.r.t. OFDM, the use of FMT with the shortest possible length pulses,-namely FMT whose pulses have length $N$ samples,-jointly with single tap sub-channel equalization is considered. It can be obtained from the general MC system model in Figure 1, by simply substituting the prototype pulse $h(n)=$ $g^{*}(-n)$ with an FMT orthogonal pulse having minimal length [7]. These pulses satisfy the orthogonality conditions given by the following system of equations

$$
\left[g^{(k)} * h^{(i)}\right](N n)=\delta_{n} \delta_{i-k}, \quad \forall(k, i) \in\{0, \ldots, M-1\}, \forall n \in \mathbb{Z},
$$

where $\delta_{n}$ denotes the Kronecker delta. It is worth noting that FMT uses sub-channel matched filters at the analysis stage. This implies that although FMT uses an $\mathrm{OH} \beta=N-M$, it does not suffer from a reduction of SNR as OFDM does. Now, looking at (5), it can be 
noticed that its solution is not unique, and thus the filter $g(n)$ coefficients can be parameterized with a minimal set of parameters $\theta$. Then, in order to have maximally frequency-confined pulses, the parameters $\theta$ that satisfy the minimum square error from a target pulse $H(f)$ are chosen as

$$
\arg \min \int_{-0.5}^{0.5}|G(f, \theta)-H(f)|^{2} d f
$$

where $G(f, \theta)$ is the frequency response of the prototype pulse $g(n)$ as function of the parameters $\theta$. Figure 2 shows an example of an FMT orthogonal pulse with $N=(9 / 8) M$. It has been obtained by considering a root-raised cosine target spectrum. It will be used in Section 6 to obtain numerical results. The OFDM pulse is also shown. It is interesting to note that the orthogonal pulse in Figure 2 has only $\beta$ coefficients that differ from a constant. More details regarding the filter design for FMT are reported in [7].

\section{Adaptive filter bank schemes}

Looking at (3), the signal-to-interference plus noise ratio (SINR) in the $k$-th sub-channel, for a given fading channel realization, can be expressed as follows

$$
\operatorname{SINR}^{(k)}(\beta)=\left(\frac{1}{\operatorname{SNR}^{(k)}(\beta)}+\frac{1}{\operatorname{SIR}^{(k)}(\beta)}\right)^{-1}
$$

where the SNR and the signal-to-interference ratio (SIR) are, respectively, defined as $S N R^{(k)}(\beta)=P_{U}^{(k)}(\beta) / P_{\eta}^{(k)}(\beta)$ and $\operatorname{SIR}^{(k)}(\beta)=P_{U}^{(k)}(\beta) / P_{I}^{(k)}(\beta)$. The average power of the signal of interest, the interference, and the noise are, respectively, defined as [14]

$$
\begin{aligned}
P_{U}^{(k)}(\beta) & =E\left|U^{(k)}(N n, \beta)\right|^{2} \\
= & P_{a}^{(k)}\left|H^{(k)}(\beta)\right| 2=P_{a}^{(k)} A^{2}(d)\left|\sum_{p=0}^{v-1} \alpha_{p} r_{g h}^{(k, k)}(-p)\right|^{2}, \\
P_{I}^{(k)}(\beta) & =P_{\mathrm{tot}}^{(k)}(\beta)-P_{U}^{(k)}(\beta), \\
P_{\eta}^{(k)}(\beta) & =E\left\{\left|\eta^{(k)}(N n, \beta)\right|^{2}\right\},
\end{aligned}
$$

where, $P_{a}^{(k)}$ denotes the power of the transmitted data symbols in the $k$-th sub-channel, and $E\{\}$ denotes the expectation w.r.t. time. Furthermore, in (8), the cross talk impulse response $r_{g h}^{(i, k)}(n)$ is defined as

$$
r_{g h}^{(i, k)}(n)=\left(g(n) e^{j 2 \pi i n / M}\right) *\left(h(n) e^{j 2 \pi k n / M}\right),
$$

and in (9) the total useful received power $P_{\text {tot }}{ }^{(k)}(\beta)$ can be computed as

$$
\begin{aligned}
P_{\text {tot }}^{(k)}(\beta) & =E\left\{\left|U^{(k)}(N n, \beta)+I^{(k)}(N n, \beta)\right|^{2}\right\} \\
& =\sum_{q \in \mathbb{Z}} \sum_{i=0}^{M-1} P_{a}^{(i)} A^{2}(d)\left|\sum_{p=0}^{\nu-1} \alpha_{p} r_{g h}^{(i, k)}(q N-p)\right|^{2} .
\end{aligned}
$$
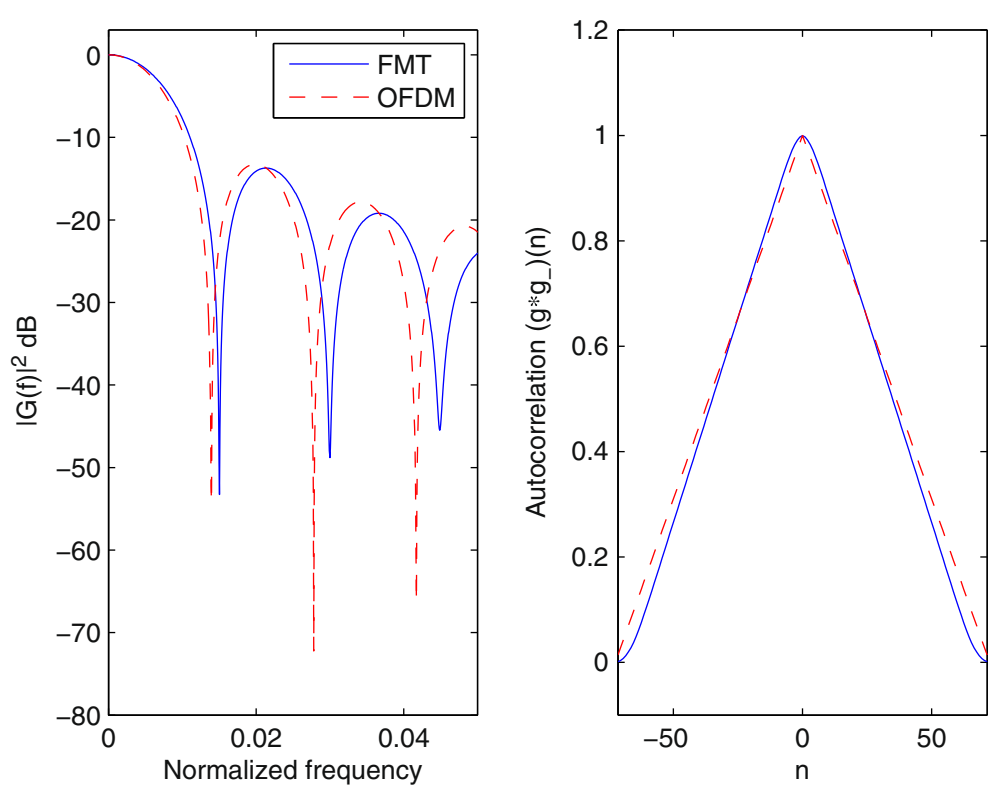

Figure 2 Frequency response and pulse autocorrelation for FMT and OFDM with parameters $N=9 / 8 M$. $M=64$. 
In the following, to facilitate the analytical description, the SINR is expressed as

$$
\operatorname{SINR}^{(k)}(\beta)=P_{a}^{(k)} \gamma^{(k)}(\beta),
$$

where, the parameter $\gamma^{(k)}(\beta)=\left|H^{(k)}(\beta)\right|^{2} /\left(P_{I}^{(k)}(\beta)+\right.$ $\left.P_{\eta}^{(k)}(\beta)\right)$ is the SINR itself when transmitting with unity power.

Now, assuming parallel Gaussian channels and statistically independent Gaussian distributed input signals-which render ISI and ICI also Gaussian (cfr. e.g., [8]) - and applying single tap zero forcing equalization, the achievable rate in bit/s for a given channel realization is defined as

$$
\begin{aligned}
C\left(\beta, \mathrm{P}_{a}, I\right) & =\frac{1}{(M+\beta) T} \sum_{k=0}^{M-1} \log _{2}\left(1+\operatorname{SINR}^{(k)}\left(\beta, \mathrm{P}_{a}, I\right)\right) \\
& =\frac{1}{(M+\beta) T} \sum_{k=0}^{M-1} \log _{2}\left(1+\frac{P_{a}^{(k)}\left|H^{(k)}(\beta)\right|^{2}}{P_{I}^{(k)}(\beta)+P_{\eta}^{(k)}(\beta)}\right),
\end{aligned}
$$

where the dependency of the SINR, and consequently of the achievable rate, on the $\mathrm{OH} \beta$, on the vector of the sub-channel transmitted powers $\mathrm{P}_{a}=\left\{P_{a}^{(k)} ; k=0, \ldots\right.$, $M-1\}$, and on the modulation scheme $I \in\{$ OFDM, FMT $\}$ has been made explicit. Therefore, the achievable rate can be maximized solving the following rate maximization (RM) problem, for a given channel realization,

$$
\begin{array}{ll}
\max _{\beta, \mathrm{P}_{a}, I} & C\left(\beta, \mathrm{P}_{a}, I\right), \\
\text { s.t. } & 0 \leq P_{a}^{(k)} \leq \bar{P}, \quad k=0, \ldots, M-1,
\end{array}
$$

where, $\bar{P}$ is the sub-channel power constraint, assumed to be constant across the sub-channels, as for example it is specified by the WLAN standard [15].

Problem (15) is solved as follows. Firstly, the optimization of the parameters for a given modulation scheme is considered. Then, the algorithm to switch between the two modulation schemes is derived.

\subsection{Adaptive OFDM}

From (15), it can be seen that the RM problem of OFDM can be solved by finding the optimal power allocation and the optimal $\mathrm{OH}$ duration, namely, the optimal $\mathrm{CP}$ of length $\beta$ samples. However, Equation (15) is not a concave optimization problem because the objective function is not concave. This can be proved by observing that the interference power depends on the transmitted powers $\mathrm{P}_{a}$. Furthermore, each term of the sum in the achievable rate formula can be expressed as a difference of log functions whose arguments depend on the elements of $\mathrm{P}_{a}$. Since the $\log$ function is a concave function and the difference of two concave/convex functions is not in general a concave/ convex function (cfr. [12] \$3.2), the achievable rate is not a concave function of $\mathrm{P}_{a}$, in general. For the same reason, the achievable rate is not a concave function of $\beta$, in general.

Since the achievable rate is neither concave in $\mathrm{P}_{a}$ nor in $\beta$, the optimal solution would require an exhaustive search, which is unfeasible.

To solve (15), it is proposed to proceed as follows.

Firstly, it is assumed to separately consider the power allocation and the CP optimization, namely, the optimal power allocation is computed for each $\mathrm{CP}$ value. Note that this assumption does not exclude any solution. Secondly, for each $\mathrm{CP}$ value, it is supposed that the interference is independent of the transmitted power. This assumption is reasonable if the interference is much smaller than the noise level, so that the SINR can be approximated by the SNR (see Equation 7). Under this assumption, the RM problem becomes concave and its solution can be found imposing the KKT conditions [12]. In particular, observing that the system is assumed to be constrained by a power spectral density (PSD) mask, it can be easily shown that the RM problem is solved by the uniform power allocation at the PSD mask, i.e., $P_{a}^{(k)}=\bar{P}, \quad k=0, \ldots, M-1$, (cfr. e.g., [16]).

Now, to optimize the CP duration $\beta$, it is observed that the CP of OFDM does not need to be as long as the channel duration to maximize the achievable rate, this is because: (a) the presence of the CP causes a loss in SNR of a factor $\beta /(M+\beta)$ since the prototype pulses at the transmitter and at the receiver are not matched, (b) the level of interference can be increased nearly up to the noise power level without reducing the achievable rate (see Equation 14), (c) the increase of $\mathrm{OH}$ causes a decrease of achievable rate by the term $1 /(M+\beta)$ (see Equation 14).

Therefore, the optimal CP duration can be found, for each channel realization, maximizing the achievable rate (14) with the uniform power allocation at the PSD mask, i.e.,

$$
\beta_{\text {opt }}=\underset{0 \leq \beta \leq v}{\arg \max }\{C(\beta)\} .
$$

Since the argument of (16) is not a concave function of $\beta$, the implementation of the optimal approach to adapt the CP to the channel realization requires an exhaustive search which is complex. Furthermore, since the WLAN channel is time variant, adaptation should be performed at each new channel realization, making the approach impractical.

A significant simplification that assures the feasibility of the $\mathrm{CP}$ adaptation, is to pre-compute a limited 
amount of $\mathrm{CP}$ values, and then adapt the $\mathrm{CP}$ over this small set of values.

To determine the limited set of CP values, in [9], it is proposed an approach based on the evaluation of the cumulative distribution function (CDF) of the achievable rate-optimal CP (16). In particular, in [9], it is shown (see also the numerical results in Section 6) that for a fixed level of noise, the achievable rate-optimal CP value depends on the specific channel realization and therefore, in general, it relies on the channel class and on the distance between the transmitter and the receiver. However, the optimal $\mathrm{CP}$ value variations are more pronounced among classes than within a given class. That is, the variation of the $\mathrm{CP}$ for the channel realizations of a given class for several distances is not as significant as if channels from different classes were drawn. Hence, in [9] it is proposed to choose a single value of $\mathrm{CP}$ for all channel realizations that belong to a certain channel class. For a given class and distance, the specific $C P$ length is chosen to be the value of $\beta$ for which the CDF of (16) is the 99-th percentile. Then, to obtain a single $\mathrm{CP}$ value associated to that class, the largest $\mathrm{CP}$ among those obtained for the considered set of distances is chosen. In particular, the considered set of distances ranges between $3 \mathrm{~m}$ and $60 \mathrm{~m}$, which are plausible values for indoor WLAN applications. The set of CP values for the five classes is then denoted with $\mathbb{P}=\left\{\beta_{B}^{(99 \%)}, \beta_{C}^{(99 \%)}, \beta_{D}^{(99 \%)}, \beta_{E}^{(99 \%)}, \beta_{F}^{(99 \%)}\right\}$. It is worth noting that the set of $\mathrm{CP}$ values would not change increasing the range of considered distances. In fact, each $\mathrm{CP}$ value is given by the correspondent smallest value of considered distances. This is because, the shorter the distance, the smaller the channel attenuation is. Consequently, the interference is high and it requires a long $\mathrm{CP}$ to be mitigated.

Clearly, once the devices know the scenario in which they are working, or equivalently the experienced channel class, the $\mathrm{CP}$ adaptation reduces to pick the corresponding value of $C P$ from the set $\mathbb{P}$. In this respect, it is worth noting that the channel class can be found from a channel estimate. In fact, since each channel class is characterized by an average rms delay spread value, we may compute the channel rms delay spread and then choose the class that has the closest average rms delay spread.

Observations about the noise power level. An important parameter to consider when designing the $\mathrm{CP}$ is the noise power level. This can simply be understood looking at (14). In fact, the optimal CP depends not only on the channel, but also on the power of the noise. This is because the interference power can be increased, without a reduction of achievable rate, nearly up to the level of the noise power. Therefore, the higher the noise level the shorter is the $\mathrm{CP}$ duration that maximizes the achievable rate. Clearly, the receiver has to keep track of the noise fluctuations, and this can be done by periodically sensing the channel in the idle periods, e.g., in the short inter frame spacing which is present in the IEEE 802.11 MAC protocol between two consecutive transmissions (cfr. [15], $\mathbb{9}$ 9.2.3).

\subsection{Adaptive FMT}

The optimal power allocation and $\mathrm{OH}$ duration for FMT can be found as done for A-OFDM. In particular, assuming a constraint on the PSD mask and the interference term independent on the transmitted power, the power allocation that maximizes the achievable rate is the one given by the same PSD limit level. Furthermore, the $\mathrm{OH}$ can be adapted to the channel condition to maximize the achievable rate, see (14). In principle, adaptation can also provide gains in FMT if we do not consider complexity. For instance with very long root-raised cosine pulses, the adaptation of the roll-off factor of the pulse and the interpolation factor $N$ of the system allows for increasing performance [13]. However, in this article, we are interested in considering an orthogonal FMT system with the shortest possible pulse, namely a pulse of length $N$ samples. In such a case, and for the considered channel model, numerical results have shown that [13] FMT with adaptive $\mathrm{OH}$ does not significantly improve the achievable rate, and in general the minimal length pulse that achieves the highest achievable rate is the one with $N=(9 / 8) M$. It is reported in Figure 2 .

\section{Hybrid FMT for achievable rate improvements}

As discussed in the previous section, the power allocation and the $\mathrm{OH}$ duration can be adapted to the channel condition to maximize the achievable rate. Beside the previous parameters, in this section, the problem of adapting the modulation scheme to the channel condition is considered. In particular, the hybrid-FMT $(\mathrm{H}-$ FMT) scheme that, depending on the channel condition, switches between A-OFDM and FMT to maximize the achievable rate is presented. The use of H-FMT is motivated as follows.

In general, thanks to the use of the CP, A-OFDM experiences an interference level lower than both the interference level of FMT with minimal length pulses and the noise level. Therefore, in general, the achievable rate of A-OFDM is limited by the power level of the noise (14). Furthermore, as previously explained, in A-OFDM the analysis and the synthesis pulses are not matched. This causes a loss in SNR of a factor $\beta /(\mathrm{M}+\beta)$ w.r.t. FMT. Now, if the system is in presence of high channel attenuation, i.e., a channel attenuation that leads to an interference power level (9) for FMT lower than the noise power 
level (10), its SINR can be approximated with the SNR (i.e. the level of the interference power is negligible compared to the level of noise power). In such a case, assuming the $\mathrm{OH}$ of FMT shorter or equal to the CP of A-OFDM, the use of FMT gives a higher value of achievable rate (14) than the one obtained with A-OFDM since it experiences an SNR higher than that of A-OFDM. Therefore, it is better to use FMT. In the other case, namely when the interference power level of FMT is higher than the noise power level, it is better to use A-OFDM.

\subsection{Implementation}

H-FMT can be implemented using the efficient implementation of a DFT modulated filter bank proposed in [11].

As depicted in Figure 3, the data signals $a^{(k)}(\mathrm{Nn})$ are processed by an $M$ points IDFT. The $M$ output streams are processed by the cyclic extension block, then are multiplied by the coefficients of the prototype pulse $g(n)$, with $n=0, \ldots, N-1$. Finally, the outputs are multiplexed with an $N$ channels parallel-to-serial converter.

The receiver comprises the following operations: the received signal $y(n)$ is demultiplexed with an $N$ channel serial-to-parallel converter, then, depending on the used modulation scheme, namely FMT or A-OFDM, the output signals are multiplied by the corresponding prototype analysis pulse coefficients $h(-n)$ with $n=0, \ldots, N-1$. The obtained streams are processed through the periodic repetition block with period $M$ [7]. Finally, the $M$-point DFT is performed. Note that the A-OFDM analysis pulse has length $M$ which is shorter than the pulse used for the synthesis stage with length $N$, but it can be considered with length $N$ simply padding $\beta$ zeros.

The choice regarding the use of either A-OFDM or FMT is made as follows.

The optimal criterion for switching between A-OFDM and FMT, for each channel realization, targets the achievable RM (15). Nevertheless, this criterion is not desirable for practical systems where the channel conditions can quickly change.

A sub-optimal criterion can be based on the estimation of the mean SNR. The idea is the following.

First of all, an estimation of the average SIR for the considered channel model can be done offline for both systems, namely for A-OFDM and FMT. Once the average SIR is known, the parameter that accounts for the $\mathrm{RM}$ and thus for the choice of the modulation to be used is the experienced SNR (see (7) and (14)). In particular, the modulation that experiences the highest minimum value between the average SIR and the SNR will be the one that allows the system to achieve the highest rate, and thus it will be selected. The SNR estimation can be done by sending a known training sequence of symbols using OFDM with a conservative long CP. In this case, the received signal results free of interference and the SINR (7) corresponds to the SNR. To adapt the system to the channel condition, the SNR estimation can be done periodically. Note that, the SNR of FMT can be computed from that of OFDM by taking into account the gain given by the use of matched pulses, i.e., by multiplying the SNR of OFDM by the quantity $\left(M+\beta_{\text {OFDM }}\right) / M$.

Now, with the specific deployed pulses and channel model, numerical results- (see Section 6)-show that the average level of the SIR experienced by FMT and A-OFDM is of about 38 and $80 \mathrm{~dB}$, respectively (these values are obtained averaging across the $M$ subchannels, and 10,000 channel realizations drawn from different channel classes considering the range of distances between 3 and $60 \mathrm{~m}$ ). Therefore, the simplified switching criterion selects FMT if the estimated mean SNR (SNR averaged across sub-channels) is below the average SIR of FMT. Otherwise A-OFDM is selected. Accordingly, the TX and RX controllers set the corresponding coefficients $g(n)$ and $h(n)$ at the transmitter and at the receiver side.

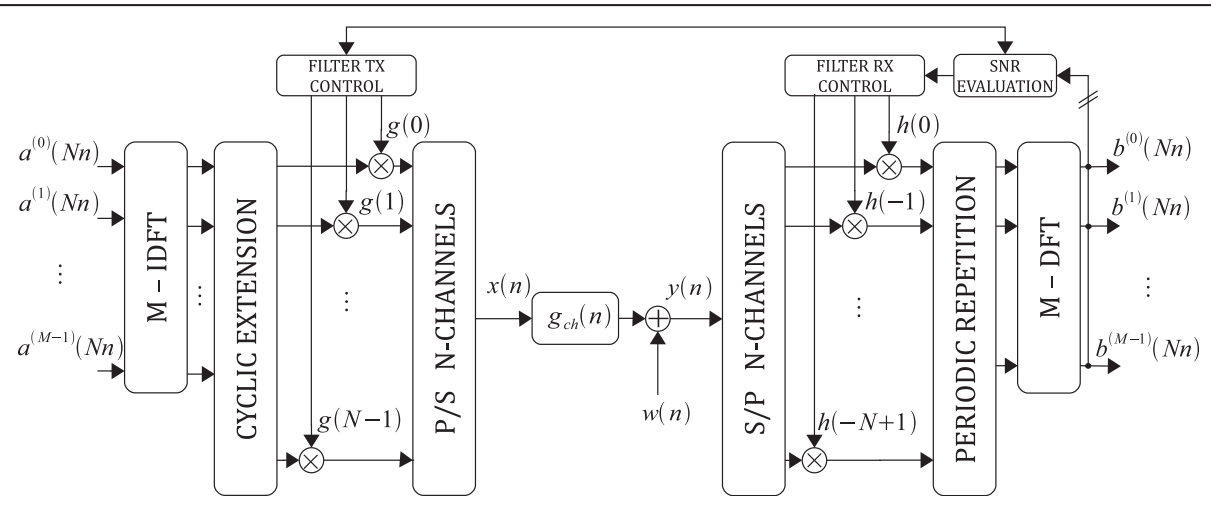

Figure 3 Hybrid FMT implementation. 
As it will be shown in the numerical results section, the criterion based on the estimation of the mean SNR gives results that are close to that given by the optimal one.

\subsection{Implementation complexity}

Taking into account that in FMT only $\beta$ coefficients of the prototype pulse differ from a constant [7], the complexity of this scheme is $(\chi M \log M+\beta) / N$ and $(\chi M \log$ $M+2 \beta) / N$ operations per sample for the synthesis and the analysis stages respectively, where $X$ depends on the FFT implementation. Hence, the complexity of H-FMT is almost the same of the efficient implementation of OFDM which is $(\chi M \log M) / N$ operations per sample for both synthesis and analysis stages. As an example, for a system with $X=1.2, M=64$, and $N=80$, the computational complexity of FMT is equal to $\{5.96,6.16\}$ op./samp. for the synthesis and the analysis stages, respectively, and the one of OFDM is equal to $5.76 \mathrm{op}$./samp. for both the synthesis and the analysis stages.

\section{Hybrid FMT for power saving}

In this section, the problem of minimizing the power transmitted by H-FMT when setting a constraint on the achievable rate is treated. To this end, in Section 5.1, the PM problem for a general MC scheme is presented. Then, in Section 5.2, its application to H-FMT is considered.

\subsection{Power minimization problem}

The PM problem refers to the minimization of the total transmitted power under a constraint on the subchannel power and a constraint on the achievable rate. It can be formulated as follows.

$$
\begin{array}{ll}
\min _{\mathrm{p}_{a}} & \sum_{k=0}^{M-1} P_{a}^{(k)}, \\
\text { s.t. } & 0 \leq P_{a}^{(k)} \leq \bar{P}, \quad k=0, \ldots, M-1, \\
& \frac{1}{N T} \sum_{k=0}^{M-1} \log _{2}\left(1+P_{a}^{(k)} \gamma^{(k)}(\beta)\right)=R,
\end{array}
$$

where $R$ denotes the target achievable rate in bit/s, $\mathrm{P}_{a}=\left\{P_{a}^{(k)}, k=0, \ldots, M-1\right\}$ is the vector of the subchannel transmitted powers, and $\bar{P}$ is the sub-channel power constraint, assumed to be constant across the sub-channels. When no constraint is given on the transmitted power, the second line of (17) reduces to $P_{a}^{(k)} \geq 0$, or equivalently $\bar{P}=\infty$.

In general, problem (17) is not convex because the interference terms in $\gamma^{(k)}(\beta)$ (see Equation 13) are dependent on the transmitted powers. A solution to
(17) could be found doing and exhaustive search on $\mathrm{P}_{a}$. However, even assuming to quantize the sub-channel power in a finite number $L$ of levels, the exhaustive search would have a complexity $O\left(L^{M}\right)$, which renders this procedure practically unfeasible.

To reduce the complexity, the interference terms are considered independent on the transmitted powers. Consequently, the problem becomes convex [12]. Therefore, if the optimal solution exists, ${ }^{\mathrm{b}}$ it satisfies the KKT conditions, and it is given by [16]

$$
P_{a}^{(k)}=P_{a}^{(k)}(\xi)=\left[\xi-1 / \gamma^{(k)}(\beta)\right]_{0}^{\bar{P}},
$$

where

$$
[x]_{a}^{b}= \begin{cases}b, & x \geq b \\ x, & a<x<b \\ a, & x \leq a,\end{cases}
$$

and $\xi$ can be computed by replacing the computed power allocation (18) in the third line of (17) [16], namely,

$$
\frac{1}{N T} \sum_{k=0}^{M-1} \log _{2}\left(1+P_{a}^{(k)}(\xi) \gamma^{(k)}(\beta)\right)=R .
$$

Relation (18) shows a typical water-filling power distribution, i.e., more power is allocated in those subchannels that experience higher normalized SNR: $\gamma^{(k)}(\beta)$.

\subsection{Power minimization algorithm for H-FMT}

The application of (18) and (20) to H-FMT can be made feasible by using the selection criteria that have been proposed in the previous section for the achievable rate maximization. The reason behind it, is that, if the interference is considered independent on the transmitted power, the PM problem can be seen as the dual problem of the RM problem [16], according to which the selection criteria for H-FMT have been designed. Clearly, due to the presence of the interference, these criteria will be sub-optimal but they are significantly more simple compared to the optimal criterion that chooses the modulation solving the non convex PM problem.

Now, once the system has selected the coefficients $g(n)$ and it has allocated the sub-channel power according to (18), the interferences experienced across the sub-channels might change. Since the coefficients $\gamma^{(k)(n)}(\beta)$ are dependent on the interference, the corresponding achievable rate may also differ from the target rate. To solve this problem, it is proposed to iteratively compute (17) until the rate target $R$ is reached. In particular, at the first iteration, the transmitted powers are set equal to the ones given by the PSD limit level specified by the WLAN standard [15]. In such a case, the interference is maximal and the 
corresponding $\gamma^{(k)(n)}(\beta)$ are the smallest. The following pseudo code summarizes the PM algorithm for $\mathrm{H}$ FMT.

(1) Set the modulation Selection Criterion. Namely, the optimal or the SNR based switching criterion.

(2) Select the system to be used (FMT or A-OFDM).

(3) Compute the coefficients $\gamma^{(k)}(\beta)$ for $k=0, \ldots, M-1$, fixing the transmitted power at the level given by the PSD mask constraint. Also compute the rate achievable with this power distribution, i.e., $C$.

(4) Set the value for the target rate $R . R \leq C$.

Se $R_{A}$ t equal to 0 .

Set the maximum number of iteration $N_{i t}$ and it $=0$. Set the desired precision $\epsilon \in[0,1]$. When showing numerical results we set $\epsilon=0.99$.

(5) while $R_{\mathrm{A}} \notin[\epsilon R,(2-\epsilon) R]$ and it $\leq N_{i t}$

Compute the optimal power allocation (18), and the corresponding coefficients $\gamma^{(k)}(\beta)$ for $k=0, \ldots$, $M-1$. Compute the SINR with (13) and the corresponding achievable rate $R_{\mathrm{A}}$ with (14).

Set it $=i t+1$.

end while

From numerical results, it is noticeable that the proposed algorithm converges in less than ten iterations either when a sub-channel power constraint is imposed or when it is not.

\section{Numerical results}

This section presents and discusses numerical results that illustrate the performance of A-OFDM, FMT, and $\mathrm{H}$-FMT when the RM and the PM problems are considered.

The following system parameters are assumed. They essentially are those of the IEEE $802.11 \mathrm{~g}$ standard [15].

The MC system uses $M=64$ sub-channels with a transmission bandwidth of $20 \mathrm{MHz}$. When a constraint on the PSD mask is considered, its level is set to $-53 \mathrm{dBm} / \mathrm{Hz}$. White Gaussian noise with PSD equal to -168 or $-138 \mathrm{dBm} / \mathrm{Hz}$ is added at the receiver. To compare the performance of the proposed systems, an OFDM baseline system is considered. It deploys a fixed $C P$ of $0.8 \mu \mathrm{s}(\beta=16$ samples), that is the value of CP employed by the IEEE $802.11 \mathrm{~g}$ standard.

\subsection{Achievable rate maximization}

Throughout the section, a constraint on the PSD mask is assumed. Therefore, as explained in Section 4, the transmitted power is allocated uniformly across the sub-channels at the PSD limit level.

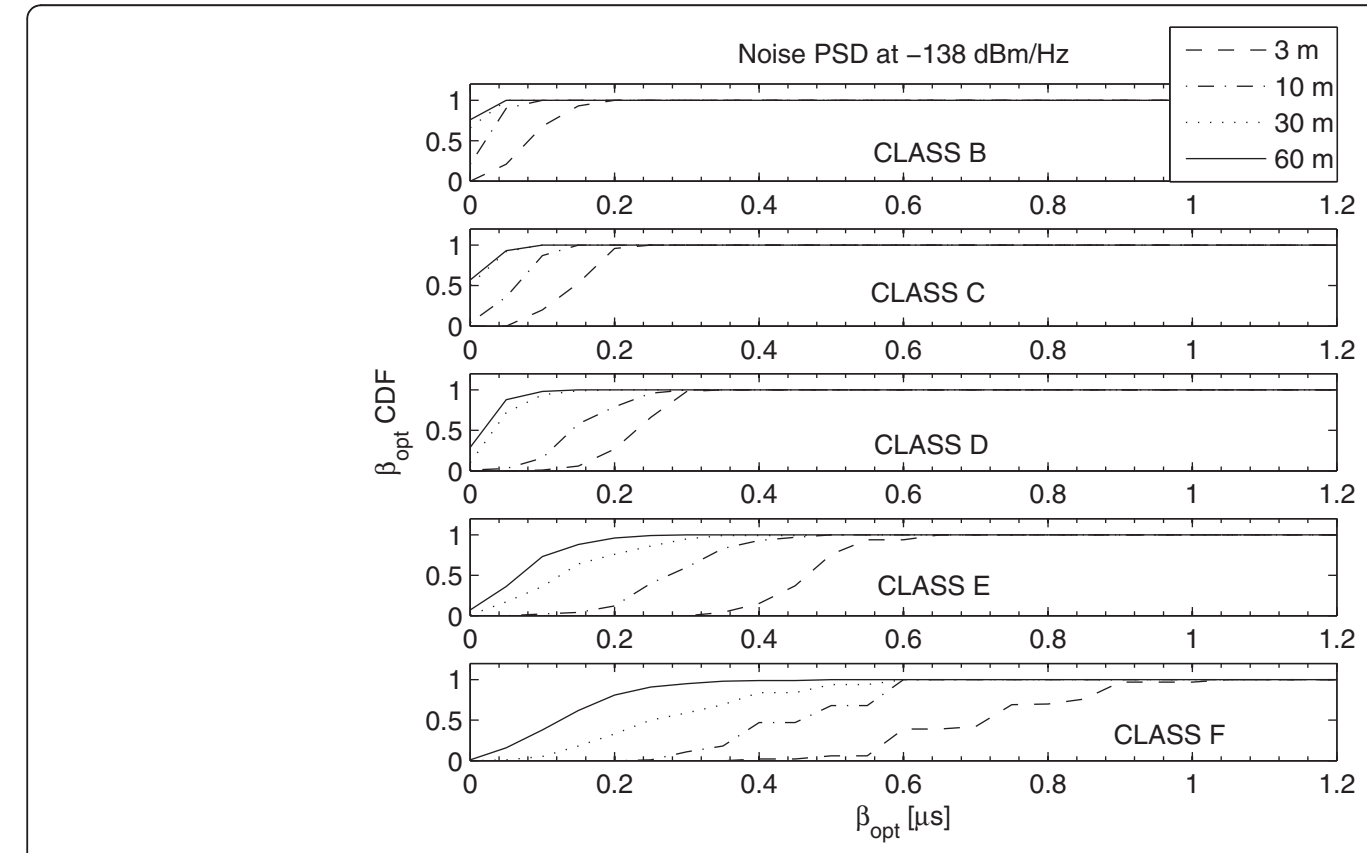

Figure 4 Optimal CP CDF for channels of class B, C, D, E, and F. The distances between transmitter and receiver are set to $3,10,30$, and $60 \mathrm{~m}$. The noise PSD equals $-138 \mathrm{dBm} / \mathrm{Hz}$. 


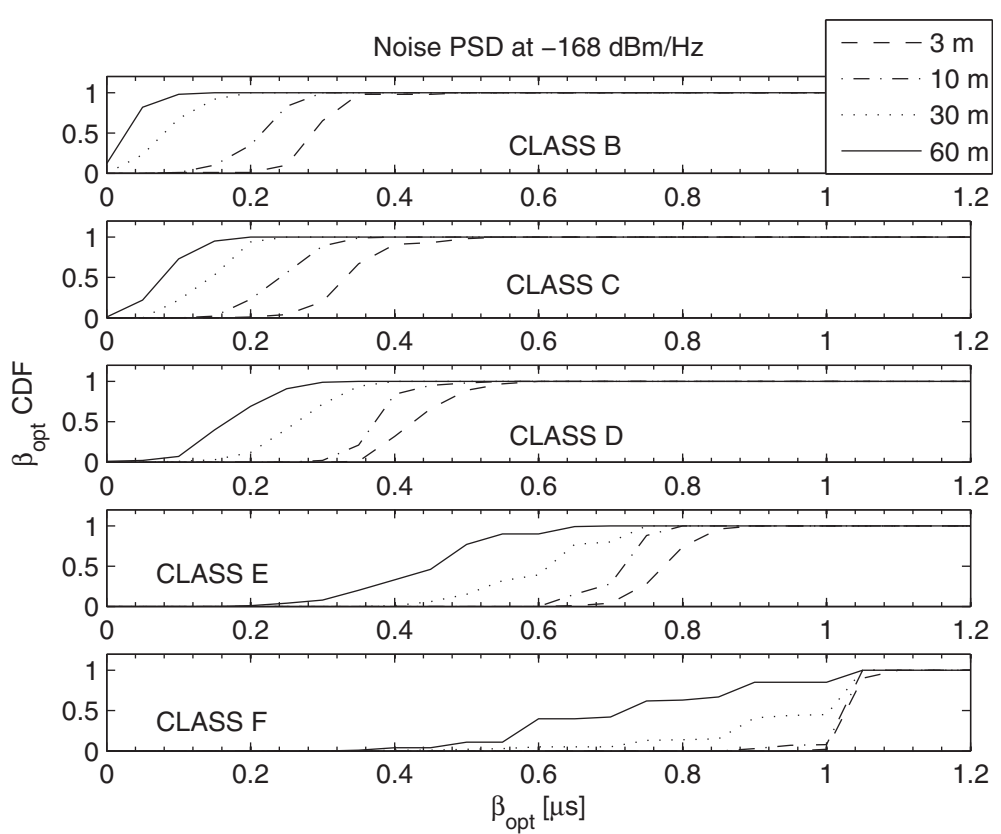

Figure 5 Optimal CP CDF for channels of class B, C, D, E, and F. The distances between transmitter and receiver are set to 3, 10, 30, and $60 \mathrm{~m}$. The noise PSD equals $-168 \mathrm{dBm} / \mathrm{Hz}$.

Figures 4 and 5 show the CDFs of the optimal CP (16) for each channel class and for different distances between transmitter and receiver when the noise PSD, respectively, equals -138 and $-168 \mathrm{dBm} / \mathrm{Hz}$. From both figures, it can be seen that the distribution of the optimal CP depends both on the distance and on the channel class. However, the dependency on the class is stronger than the one on the distance. Furthermore, the optimal CP length increases when the noise power level decreases. This, as explained in Section 3.1, is because the lower the noise power, the higher is the impact of the interference power on the achievable rate (see
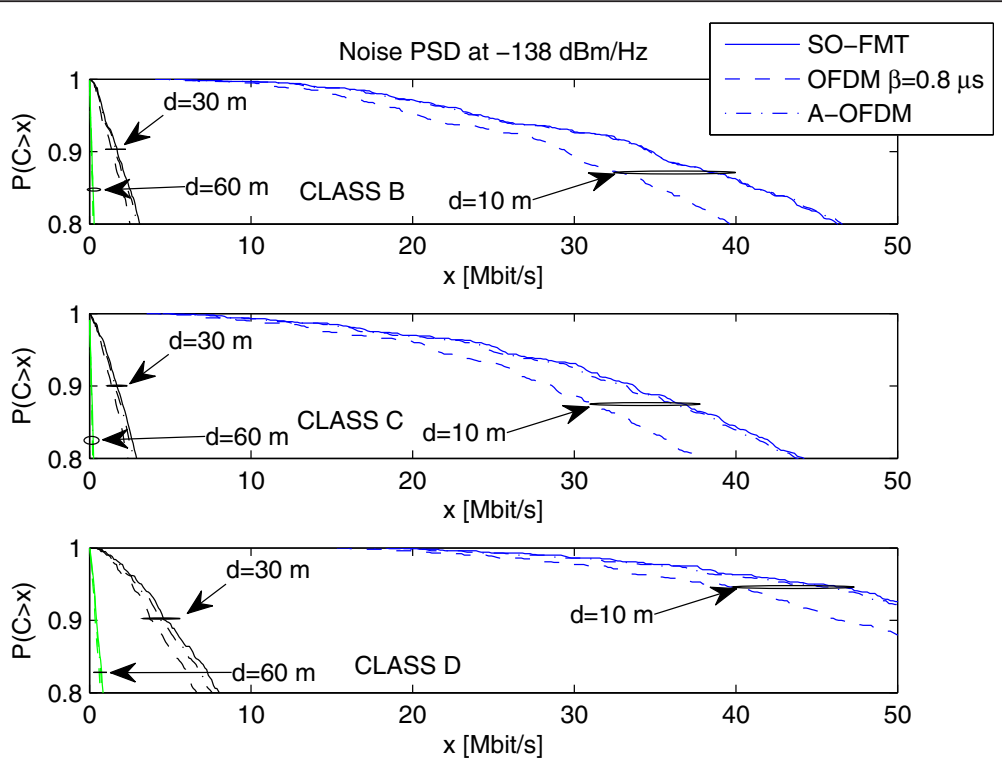

Figure 6 Achievable rate CCDF for channel classes B, C, and D obtained using FMT, A-OFDM, and OFDM with a fixed CP of $0.8 \mu \mathrm{s}$. The noise PSD equals $-138 \mathrm{dBm} / \mathrm{Hz}$. 

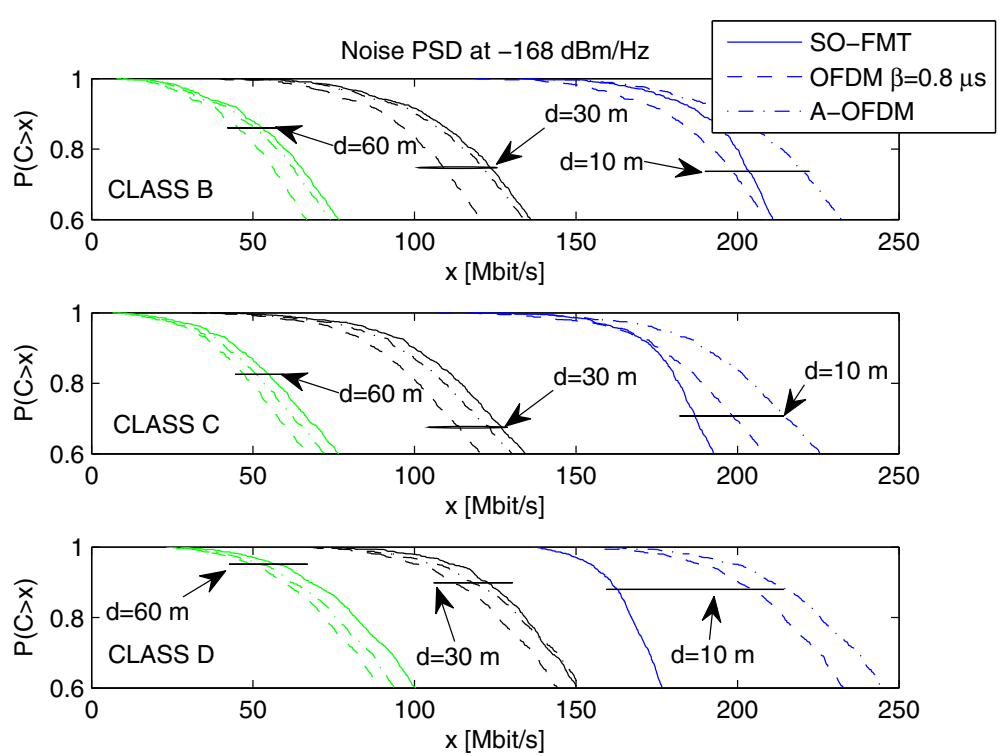

Figure 7 Achievable rate CCDF for channel classes B, C, and D obtained using FMT, A-OFDM, and OFDM with a fixed CP of $0.8 \mu$ s. The noise PSD equals $-168 \mathrm{dBm} / \mathrm{Hz}$.

Equation 14). Thus, a longer $\mathrm{CP}$ is required to mitigate this effect and maximize the achievable rate.

The sets of CPs computed according to the optimal CP CDF criterion (see Section 3.1) can be determined from Figures 4 and 5:

$$
\begin{aligned}
\mathbb{P}_{\text {NIGH }} & =\left\{\beta_{99 \%}^{B}=0.2, \beta_{99 \%}^{C}=0.24, \beta_{99 \%}^{D}=0.3, \beta_{99 \%}^{E}\right. \\
& \left.=0.65, \beta_{99 \%}^{F}=1.05\right\} \mu \mathrm{s} ;
\end{aligned}
$$

$$
\begin{aligned}
\underset{\text { NOISE }}{\mathbb{P}_{L O W}} & =\left\{\beta_{99 \%}^{B}=0.4, \beta_{99 \%}^{C}=0.5, \beta_{99 \%}^{D}=0.6, \beta_{99 \%}^{E}\right. \\
& \left.=0.9, \beta_{99 \%}^{F}=1.1\right\} \mu \mathrm{s} .
\end{aligned}
$$

Figures 6 and 7 show the complementary CDFs (CCDFs) of the achievable rate obtained using FMT, AOFDM, and the baseline system when the noise PSD is, respectively, equal to -138 and $-168 \mathrm{dBm} / \mathrm{Hz}$. For the sake of readability, we only show results for channel

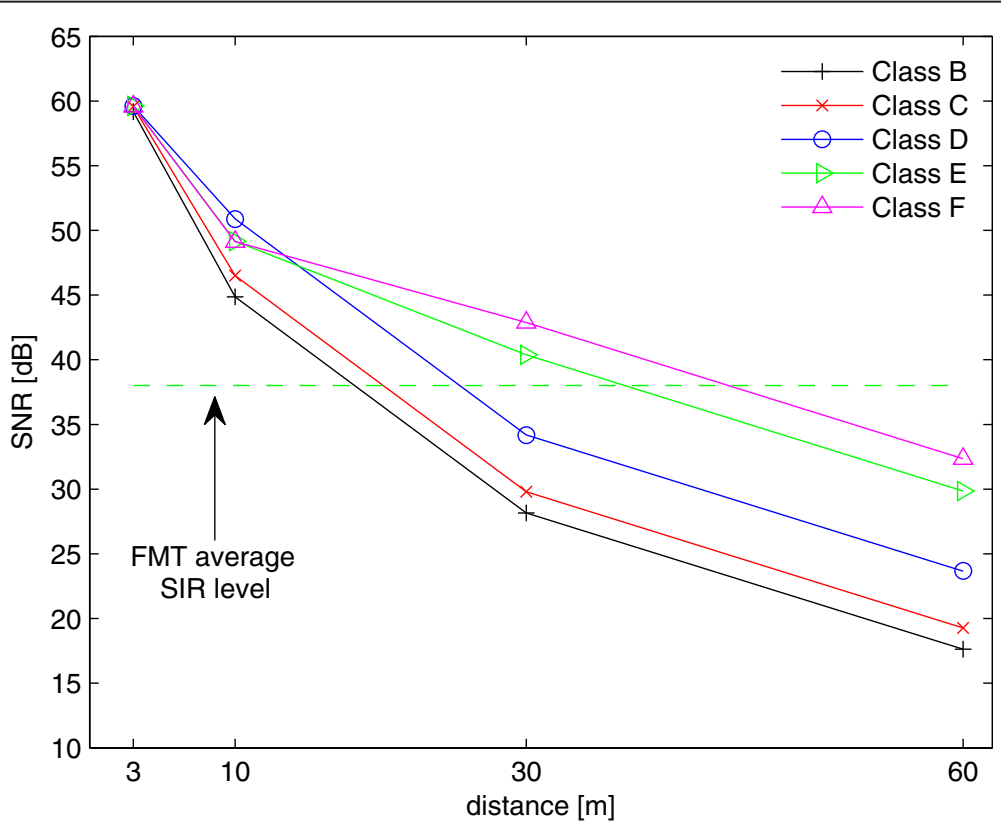

Figure 8 Mean SNR as a function of the distance and average SIR level of FMT. 


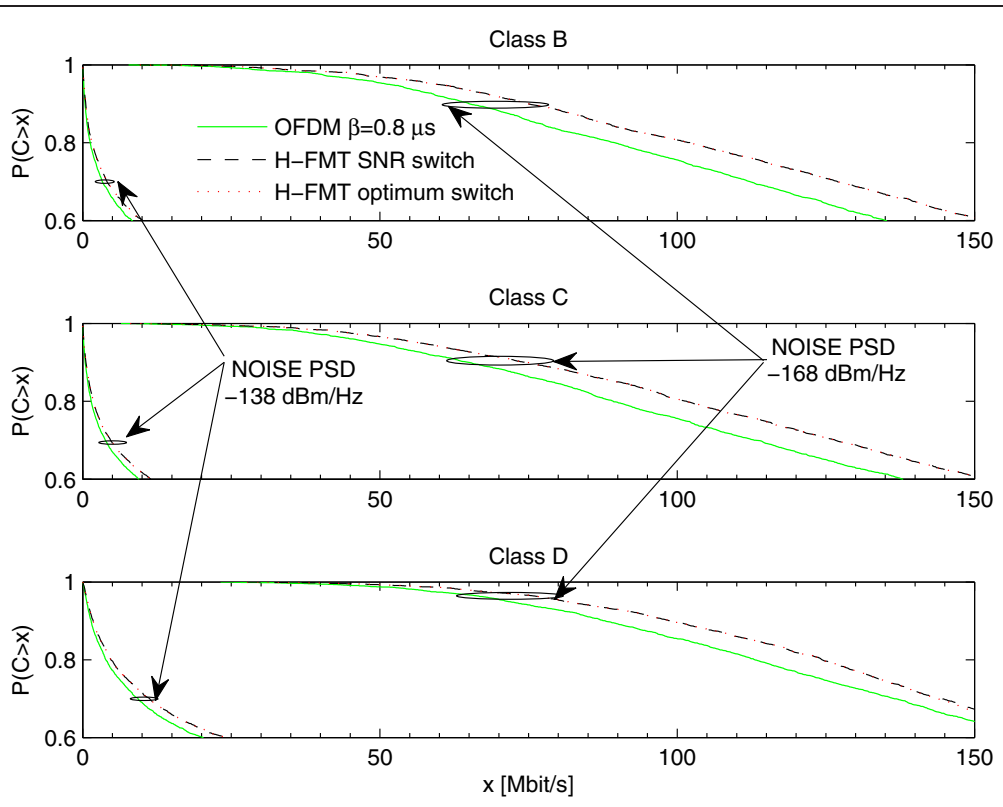

Figure 9 Achievable rate CCDF using H-FMT and OFDM with a fixed CP of $0.8 \mu$ s for channel classes B, C, and D.

classes B, C, and D and for distances between transmitter and receiver equal to 10,30 , and $60 \mathrm{~m}$.

From Figure 6, we can observe that when the noise is high it is better to use FMT than both OFDM and A-OFDM. This is also true, when the noise is low as in Figure 7, but for large values of distance $d=\{30,60\} \mathrm{m}$. Therefore, FMT shows better performance than both AOFDM and baseline OFDM in the low SNR region. On the contrary, for a short distance of $10 \mathrm{~m}, \mathrm{~A}-\mathrm{OFDM}$ is able to achieve higher rate than FMT. Although not shown, the results obtained with a distance of $3 \mathrm{~m}$ behave as the ones obtained with a distance of $10 \mathrm{~m}$.

Now, Figure 8 shows the average SNR experienced by FMT for each channel class and for distances between transmitter and receiver of $\{3,10,30,60\} \mathrm{m}$. The noise PSD equals $-168 \mathrm{dBm} / \mathrm{Hz}$. The average SNR experienced by OFDM can be obtained adding to the showed SNR a factor $10 \log _{10}(M /(M+\beta))$, e.g., for $\beta_{99 \%}^{F}=1.1 \mu \mathrm{s}$, it has to be lowered by $1.28 \mathrm{~dB}$. Figure 8 also shows the average SIR (averaged across all the channel classes and across all the distances) of FMT. It is worth noting that regarding A-OFDM, it always experiences a SIR that is several dBs higher than the one of FMT.

Figure 9 shows the achievable rate CCDF for the baseline system and for H-FMT that uses both the optimal and the mean SNR-based modulation selection criterion. The simulated channel classes are the B, C, and D. For each class, the curves are computed considering random distances from the set $\{3,10,30,60\} m$. Notably, both modulation selection criteria give the same performance. Furthermore, with probability 0.9, H-FMT provides gains in achievable rate of $\{29 \%, 23 \%, 21 \%\}$ and $\{16 \%$,
$15 \%, 12 \%\}$ w.r.t. OFDM for channel classes $\{B, C, D\}$ and for noise PSD of -138 and $-168 \mathrm{dBm} / \mathrm{Hz}$, respectively. More precisely, with probability 0.9 , for channel classes B, C, and D, OFDM exceeds $\{60,64,85\} \mathrm{Mbit} / \mathrm{s}$, while H-FMT exceeds $\{72,74,98\} \mathrm{Mbit} / \mathrm{s}$. Finally, looking at Figures 6 and 7, it should be noted that also if not explicitly highlighted before, the achievable rate CCDF of H-FMT for each distance and for each class corresponds to the right most curve.

\subsection{Power minimization}

Figures 10 and 11 show the CDFs of the average transmitted power per sub-channel, respectively, for a noise PSD of -138 and $-168 \mathrm{dBm} / \mathrm{Hz}$. They have been obtained solving the PM problem (17) for H-FMT with the proposed PM algorithm using both the optimal and the mean SNR based modulation selection criteria, and for the baseline system. The results are shown imposing a sub-channel power constraint given by a PSD mask of $-53 \mathrm{dBm} / \mathrm{Hz}$. Furthermore, the case of no subchannel power constraint is shown for H-FMT that uses the optimal modulation selection criterion. The results obtained with A-OFDM are also shown. These are meant to show the best performance obtainable using OFDM. The results are obtained drawing the channels randomly among the classes $\{B, C, D\}$, and for distances in 1-60 $\mathrm{m}$. Furthermore, for each channel realization the target rate $R$ is set equal to the achievable rate of the baseline system (OFDM with fixed CP of $0.8 \mu \mathrm{s}$ ) whose transmitted powers are given by the PSD limit level.

From Figures 10 and 11, the following observations can be made. 


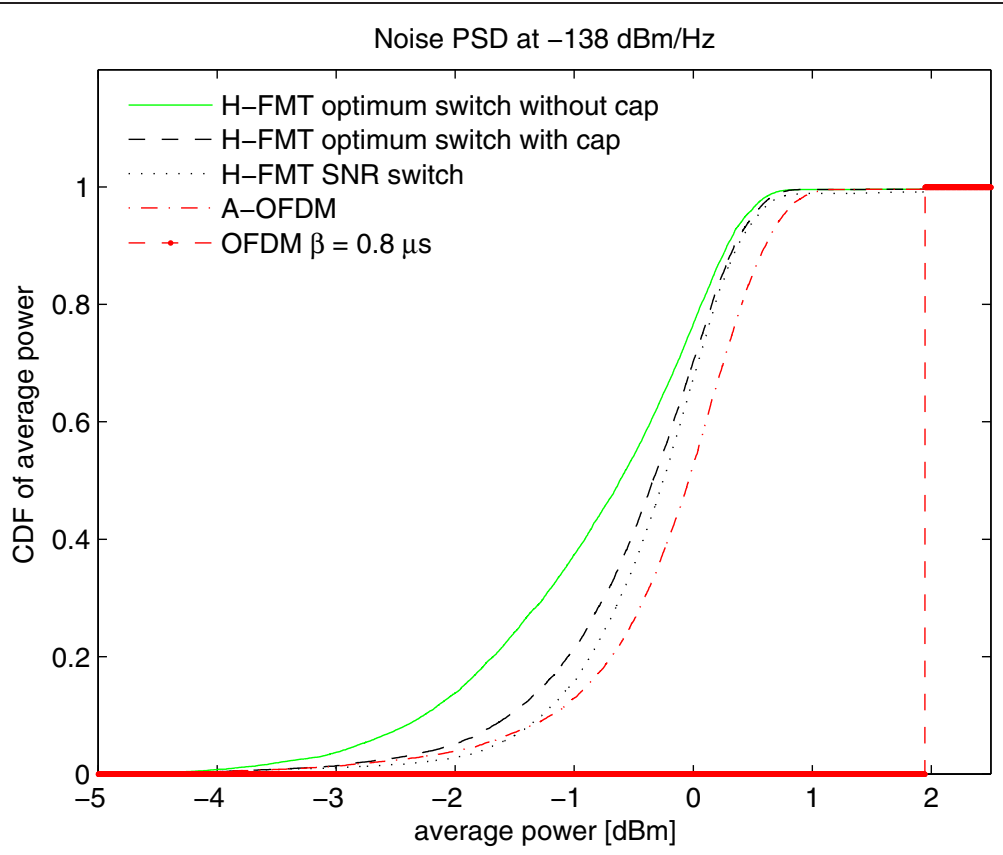

Figure $10 \mathrm{CDF}$ of the average transmitted power obtained using the proposed PM algorithm. The comparison is among H-FMT, OFDM with CP equal to $0.8 \mu \mathrm{s}$, and A-OFDM. The noise PSD equals $-138 \mathrm{dBm} / \mathrm{Hz}$.

The modulation selection criterion based on the mean SNR estimation works close to the optimal one. The power saving given by the use of H-FMT without subchannel power constraint is negligible w.r.t. the use of a sub-channel power constraint.
With probability equal to $0.9, \mathrm{H}-\mathrm{FMT}$ and A-OFDM, respectively, transmit with power of about 1.6 and $1.34 \mathrm{~dB}$ less than the baseline system when the noise PSD equals $-138 \mathrm{dBm} / \mathrm{Hz}$, and of about 1.9 and $1.4 \mathrm{~dB}$ less when the noise PSD equals $-168 \mathrm{dBm} / \mathrm{Hz}$.

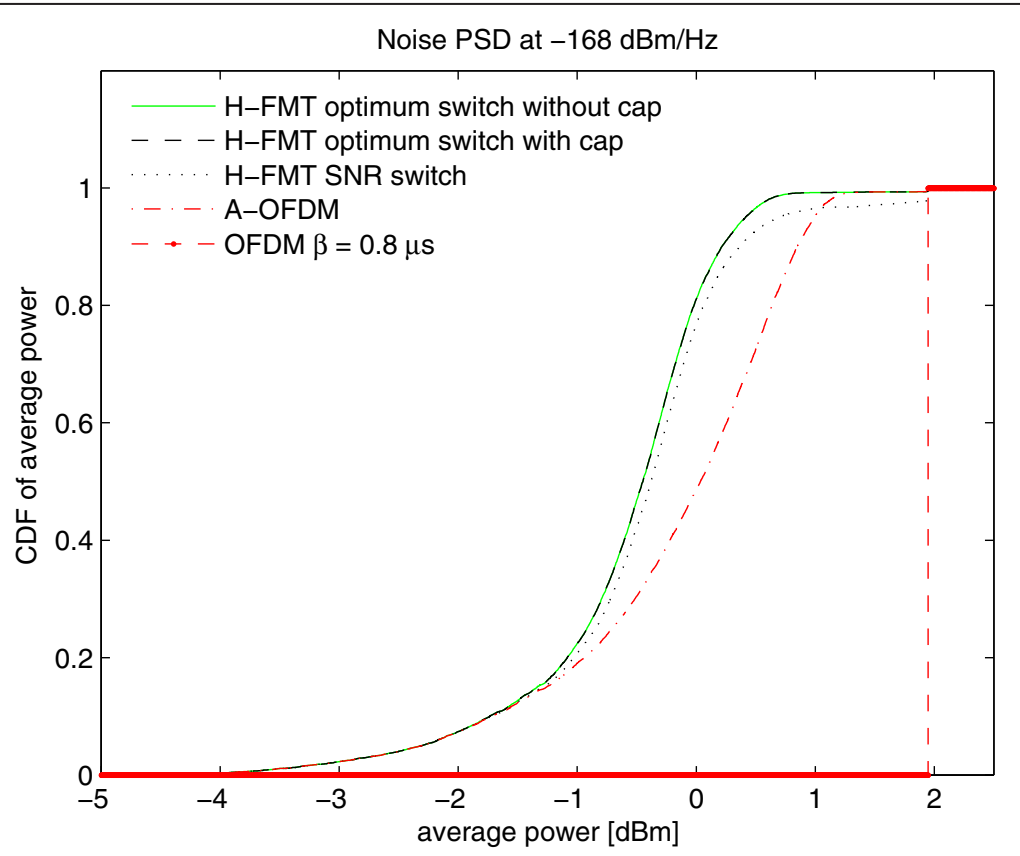

Figure $11 \mathrm{CDF}$ of the average transmitted power obtained using the proposed PM algorithm. The comparison is among H-FMT, OFDM with CP equal to $0.8 \mu \mathrm{s}$, and A-OFDM. The noise PSD equals $-168 \mathrm{dBm} / \mathrm{Hz}$. 
This translates in a power saving in the range of 30 $36 \%$ w.r.t. the baseline system. However, it is also noticeable that there are a $20 \%$ of channels where the power saving is of about $3 \mathrm{~dB}$.

\section{Conclusions}

The use of adaptive MC modulation over WLAN channels has been investigated, and a hybrid FMT architecture has been proposed. It wisely switches between adaptive OFDM and FMT with minimal length pulses to provide achievable rate or power saving gains w.r.t. conventional OFDM that deploys a fixed value of CP. Hybrid FMT can be implemented through an efficient architecture whose complexity is almost the same of OFDM. Numerical results, obtained by simulating the proposed architecture over typical WLAN channels, have shown that the proposed architecture can improve the performance of WLAN devices up to the $30 \%$ in achievable rate, or up $2 \mathrm{~dB}$ in transmitted power in $80 \%$ of channel realizations.

\section{Endnotes}

${ }^{1}$ In order to simplify the notation, through this study, the dependency of the signals on $T$ is omitted.

${ }^{2}$ Note that in some cases it is possible that the target rate is not reachable under a sub-channel power constraint.

\section{Competing interests}

The authors declare that they have no competing interests.

Received: 3 September 2012 Accepted: 3 January 2013

Published: 4 February 2013

\section{References}

1. L Cai, P Li, N Kato, R Fantacci, User cooperation in wireless networks. IEEE Wireless Commun. 19, 2 (2012)

2. S Sadr, A Anpalagan, K Raahemifar, Radio resource allocation algorithms for the downlink of multiuser OFDM communication systems. IEEE Commun. Surveys \& Tutorials 11(3), 92-106 (2009)

3. S D'Alessandro, N Moret, AM Tonello, Hybrid filtered multitone architecture for WLAN applications, in Proceedings of the IEEE Personal, Indoor, and Mobile Radio Conference (PIMRC) (Istanbul, Turkey, 2010), pp. 1076-1080.

4. G Cherubini, E Eleftheriou, S Olcer, Filtered multitone modulation for very high-speed digital subscriber lines. IEEE J. Sel. Areas Commun. 20(5), 1016-1028 (2002)

5. AM Tonello, Performance limits for filtered multitone modulation in fading channels. IEEE Trans. Wireless Commun. 4, 2121-2135 (2005)

6. AM Tonello, F Pecile, Analytical results about the robustness of FMT modulation with several prototype pulses in time-frequency selective fading channels. IEEE Trans. Wireless Commun. 7, 1634-1645 (2008)

7. N Moret, AM Tonello, Design of orthogonal filtered multitone modulation systems and comparison among efficient realizations. Eurasip J. on Advances in Signal Processing 2010(1), 18 (2010). doi:10.1155/2010/141865, ISSN 1687-6180

8. J Seoane, S Wilson, S Gelfand, Analysis of Intertone and Interblock Interference in OFDM when the Length of the Cyclic Prefix is Shorter than the Length of the Impulse Response of the Channel, in Proc. of IEEE Global Telecommun. Conf. (GLOBECOM) (Phoenix, USA, 1997), pp. 32-36. Nov

9. S D'Alessandro, AM Tonello, L Lampe, Improving WLAN Capacity via OFDMA and Cyclic Prefix Adaptation, in Proc. of IEEE IFIP Wireless Days Conference (WD) (Paris, France, 2009), pp. 1-5. Dec

10. V Ercegl, L Shumacher et al., doc.: IEEE 802.11-03/940r4, in IEEE P802.11 Wireless LANs, TGn Channel Models. (IEEE 802.11 documents, 2013) https://mentor.ieee.org/802.11/documents?is_dcn=940\&is_year=2003, Accessed 29 January 2013

11. AM Tonello, Time Domain and Frequency Domain Implementations of FMT Modulation Architectures, in Proc. of IEEE Int. Conf. on Acoustic, Speech, and Signal Processing (ICASSP) (Toulose, France, 2006). May

12. S Boyd, L Vandenberghe, Convex Optimization (Cambridge University Press, Cambridge, UK, 2009)

13. N Moret, S D'Alessandro, AM Tonello, Performance of FMT with Time Confined and Frequency Confined Pulses over Indoor Radio Channels, in proc. of IEEE IFIP Wireless Days (WD 2010) (Venice, Italy, 2010), pp. 1-5. Oct

14. AM Tonello, S D'Alessandro, L Lampe, Cyclic prefix design and allocation in Bit-loaded OFDM over power line communication channels. IEEE Transactions on Communications 58(11), 3265-3276 (2010)

15. IEEE Computer Society, 802.11 Standard, Wireless LAN Medium Access Control and Physical Layer Specification (IEEE, New York, USA, 2007)

16. N Papandreou, T Antonakopoulos, Bit and power allocation in constrained multi-carrier systems: the single-user case. EURASIP J. on Adv. in Sig. Processing 2008, Article ID 643081 (2008)

doi:10.1186/1687-1499-2013-22

Cite this article as: D'Alessandro and Tonello: Rate improvements and power saving with a hybrid filtered multitone scheme. EURASIP Journal on Wireless Communications and Networking 2013 2013:22.

\section{Submit your manuscript to a SpringerOpen ${ }^{\odot}$ journal and benefit from:}

- Convenient online submission

- Rigorous peer review

- Immediate publication on acceptance

- Open access: articles freely available online

- High visibility within the field

- Retaining the copyright to your article

Submit your next manuscript at $>$ springeropen.com 